J. Clin. Chem. Clin. Biochem.

Vol. 24, 1986, pp. 637-639

(C) 1986 by Walter de Gruyter \& Co. Berlin - New York

\title{
Renale Ausscheidung von Gesamtporphyrinen und Hippursäure bei der Ratte
}

\author{
Von J. Gartzke und D. Burck \\ Zentralinstitut für Arbeitsmedizin der DDR, Berlin (Direktor: OMR Prof. Dr. sc. med. H. Kreibich) Direktions- \\ bereich Arbeitshygiene (Leiter: OMR Doz. Dr. sc. med. K. Ruppe)
}

(Received March 25, 1986)

Zusammenfassung: Die von adulten männlichen Wistar-Ratten mit dem Urin ausgeschiedenen Mengen an Gesamtporphyrinen, Hippursäure und Kreatinin zeigten für Hippursäure und Kreatinin eine weitgehend normale, für die Porphyrinausscheidung dagegen eine bimodale Verteilung. Die Verteilung der Porphyrinausscheidung blieb auch bei Verwendung von Kreatinin als Bezugsgröße erhalten. Deshalb sollten Ratten bei biochemischen und/oder toxikologischen Experimenten zuvor auf ihre Homogenität in bezug auf die zu bestimmenden Kenngrößen geprüft werden.

\section{Renal excretion of total porphyrins and hippuric acid in rats}

Summary: The amounts of total porphyrins, hippuric acid and creatinine, excreted in urine by adult male Wistar rats, exhibited normal distributions for hippuric acid and creatinine, but a bimodal distribution for total porphyrins. This typical distribution of total porphyrins was still observed when creatinine was used as reference parameter. In biochemical and toxicological experiments in rats, the tested parameters should be therefore be investigated for homogeneity.

\section{Einführung}

Tierexperimentelle Untersuchungen zu Veränderungen der Leberfunktion unter Einwirkung von Xenobiotika erfordern ein in bezug auf die zur Untersuchung gewählten biochemischen Kenngrößen homogenes Tiermaterial, um das Erkennen schwach ausgeprägter, biochemischer Effekte statistisch objektivieren zu können. Chronische Versuche zur Wirkung eines Noxeneinflusses erfordern, insbesondere bei kleinen Versuchstieren, die Bestimmung möglichst nichtinvasiver und wiederholbarer biochemischer Kenngrößen. Deshalb ist in solchen Fällen die Bestimmung adäquater Kenngrößen im Urin vorteilhaft.

Porphyrine im Urin sind Ausdruck ihrer Synthese in der Leber (1). Als Vorstufen wichtiger, für den Xenobiotikametabolismus in der Leber verantwortlicher Hämenzyme besitzen die Porphyrine wesent- liche Bedeutung. Die Hippursäureausscheidung bekannt als Leberfunktionstest nach Quick (2) erfaßt die Glycinkonjugationsleistung der Leber mit dem Xenobiotikum bzw. dessen Metaboliten. Die Hippursäure ist z. B. der Hauptmetabolit des arbeitsmedizinisch relevanten Schadstoffes Toluol.

\section{Material und Methoden}

Es wurde die renale Ausscheidung von Gesamtporphyrinen, Hippursäure und Kreatinin bei adulten, männlichen WistarRatten (Shoe Wistar C) bestimmt. Die Tierhaltung war konventionell in Käfigen mit einer Populationsdichte von $\mathrm{N}=10$, die Fütterung erfolgte mit Standardfutter (Pellets, VEB KIM Mischfutterwerk GDR; Bleigehalt $0,3 \mathrm{mg} / \mathrm{kg}$, Quecksilber 30 $\mu \mathrm{g} / \mathrm{kg}$, Cadmium $260 \mu \mathrm{g} / \mathrm{kg}$ ) und Wasser ad libitum. Die aus experimentellen Gründen gewählte Urinsammlung von 18 Stunden (13.00-7.00 Uhr) erfolgte im Dunkeln nach oraler Gabe von $30 \mathrm{ml}$ Wasser pro kg Körpergewicht ohne Nahrungs- und Flüssigkeitszufuhr. Zur Stabilisierung der Porphyrine wurde 
der Urin über Natriumbicarbonat $(0,11 \mathrm{~g})$ gesammelt. Die derivativspektroskopische Bestimmung der Gesamtporphyrine erfolgte nach Ansäuern von $1 \mathrm{ml}$ zentrifugiertem Ürin mit $0,1 \mathrm{ml}$ $5 \mathrm{~mol} / \mathrm{l}$ Salzsäure und Messen des spektralen Verlaufs zwischen 370 und $440 \mathrm{~nm}$ nach dem Verfahren von Sawitzky \& Golay (3) mit dem Spektralphotometer Specord M 40 (VEB Carl Zeiss Jena) unter Verwendung der Computerkassette Data Handling I (4). Diese Bestimmung zeichnet sich neben der Praktikabilität durch eine hohe Spezifität und Sensitivität aus. Gleiches trifft auch für die angewandte dünnschichtchromatographische Hippursäurebestimmung zu (5).

Die Ermittlung des Kreatiningehaltes erfolgt wegen der möglicherweise zu falschen Ergebnissen führenden hohen Ascorbinsäuregehalte des Rattenurins mit einer kinetischen Methode (6). Die Sammlung über Natriumbicarbonat beeinflußt die Ergebnisse der Methode nicht.

\section{Ergebnisse und Diskussion}

Die zu einer Referenzwertermittlung notwendigen Auswahlkriterien und statistischen Ergebnisse sind in Tabelle 1 därgestellt.

Während sowohl die Hippursäure- (Abb. 1 a) als auch die Kreatininausscheidung (Abb. 1 b) sowie die in der Tabelle 1 angeführten Kenngrößen Körpergewicht und Diurese der untersuchten Ratten weitgehend einer normalen Verteilung entsprechen, besteht für die Porphyrinausscheidung eine signifikante bimodale Verteilung (Abb. 1 c) mit maximalen Differenzen von etwa $5 \cdot 10^{2} \mathrm{nmol} / 18$ Stunden. Diese bimodale Verteilung bleibt auch dann erhalten, wenn Kreatinin als Bezugsgröße für die Diurese verwendet wird (Abb. 1d).

Eine getrennte statistische Bewertung beider Porphyrinexkretionspeaks mit einer willkürlichen Trennung bei $6 \mathrm{nmol} / 18$ Stunden zeigt Medianwerte von 1,93 bzw. 18,9 nmol/18 Stunden (Mittelwerte: 2,20 bzw. $19,2 \mathrm{nmol} / 18$ Stunden).

Ein Altersgang der Porphyrinausscheidung war nach eigenen Untersuchungen bei adulten Tieren nicht nachweisbar, ebenso keine Inversion des Porphyrinausscheidungstyps, d. h. Niedrigausscheider blieben Niedrigausscheider, Hochausscheider blieben Hochausscheider. Die gefundene bimodale Verteilung der Porphyrinausscheidung ließ sich über einen Zeitraum von 3 Jahren nachweisen und konnte auch bei einer untersuchten kleinen Population $(\mathrm{N}=20)$ von WKY-Ratten (Wistar-Kyoto-Stamm) bestätigt werden. Ob leberspezifische, renale, genetische oder andere Effekte für die gefundene bimodale Porphyrinexkretion verantwortlich $\mathrm{zu}$ machen sind, wurde nicht geprüft. Bekannt sind jedoch genetische Polymorphismen in bezug auf den Cytochrom P 450-Gehalt der Rattenleber (7). Das könnte seine Ursache in der unterschiedlich hohen Porphyrinsynthese der Ratten-
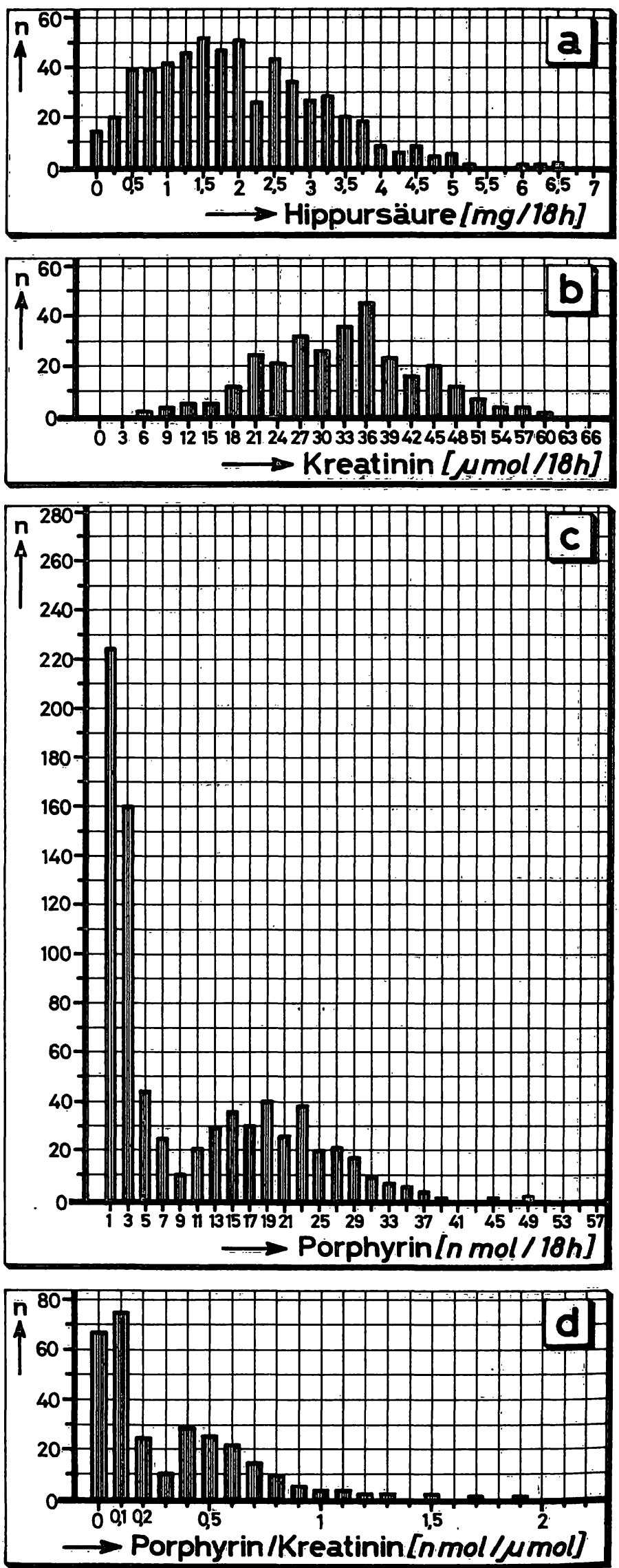

Abb. 1 a-d. Graphische Darstellung der Verteilung der renalen Ausscheidung von

a) Hippursäure (mg/18 Stunden) $\cdot(\mathrm{mg} \cdot 5,58=$ $\mu \mathrm{mol}$ )

b) Kreatinin ( $\mu \mathrm{mol} / 18 \cdot$ Stunden)

c) Gesamtporphyrineri (nmol/18 Stunden)

d) Gesamtporphyrinen/Kreatinin (nmol/umol) 
Tab. 1. Zur Referenzwertermittlung notwendige Auswahlkriterien und statistische Ergebnisse.

\begin{tabular}{|c|c|c|c|c|c|c|c|c|c|}
\hline \multirow[t]{2}{*}{ Kenngröße } & \multirow{2}{*}{$\begin{array}{l}\text { Anzahl } \\
\text { unter- } \\
\text { suchter } \\
\text { Tiere }\end{array}$} & \multirow{2}{*}{$\begin{array}{l}\text { Mittel- } \\
\text { wert }\end{array}$} & \multirow[t]{2}{*}{ Median } & \multirow{2}{*}{$\begin{array}{l}\text { Standard- } \\
\text { ab- } \\
\text { weichung }\end{array}$} & \multicolumn{2}{|c|}{ Extremwerte } & \multicolumn{2}{|c|}{$\begin{array}{l}\text { Abweichung von der } \\
\text { Normalverteilung }\end{array}$} & \multirow{2}{*}{$\begin{array}{l}\chi^{2} \text {-Wert, } \\
\text { errechnet, } \\
\text { in } 0 \\
\text { Tabellenwert } \\
\text { für } p \leqslant 0,05\end{array}$} \\
\hline & & & & & $\begin{array}{l}\text { Mini- } \\
\text { mum }\end{array}$ & $\begin{array}{l}\text { Maxi- } \\
\text { mum }\end{array}$ & Schiefe & Exzess & \\
\hline $\begin{array}{l}\text { Körpergewicht } \\
\text { (g) }\end{array}$ & 776 & 202 & 204 & $\pm 15,6$ & 166 & 270 & $-0,1526$ & 2,391 & $\begin{array}{c}64,684 \\
(22,362)\end{array}$ \\
\hline $\begin{array}{l}\text { Diurese } \\
(\mathrm{ml} / 18 \mathrm{~h})\end{array}$ & 776 & 9,1 & 9,18 & $\pm 2,43$ & 1,2 & 17,9 & $-0,1391$ & $-0,0547$ & $\begin{array}{c}12,025 \\
(18,308)\end{array}$ \\
\hline $\begin{array}{l}\text { Kreatinin } \\
(\mu \mathrm{mol} / 18 \mathrm{~h})\end{array}$ & 574 & 35,2 & 35,5 & $\pm 9,28$ & 4,42 & 72,3 & $-0,092$ & 1,163 & $\begin{array}{c}6,452 \\
(18,308)\end{array}$ \\
\hline $\begin{array}{l}\text { Hippursäure*) } \\
\text { (mg/18 h) }\end{array}$ & 582 & 1,98 & 1,83 & $\pm 1,19$ & 0,07 & 6,41 & 0,5269 & 0,2101 & $\begin{array}{c}30,576 \\
(27,570)\end{array}$ \\
\hline $\begin{array}{l}\text { Gesamtporphyrine } \\
\text { (nmol/18 h) }\end{array}$ & 766 & 9,7 & 4,07 & $\pm 9,74$ & 0,23 & 47,3 & 0,8817 & $-0,497$ & $\begin{array}{r}1508,800 \\
(40,113)\end{array}$ \\
\hline $\begin{array}{l}\text { Gesamtporphyrine/ } \\
\text { Kreatinin } \\
\text { (nmol/ } \mu \mathrm{mol})\end{array}$ & 292 & 0,313 & 0,171 & $\pm 0,337$ & 0,003 & 2,7 & 1,469 & 2,636 & $\begin{array}{l}208,640 \\
(15,508)\end{array}$ \\
\hline
\end{tabular}

*) $\mathrm{mg} \cdot 5,58=\mu \mathrm{mol}$

leber haben. Unterschiedliche Dispositionen gegenüber Xenobiotika wären die Folge, was sich beispielsweise bei der Ermittlung von Dosis-Wirkungs-Beziehungen störend bemerkbar machen würde.

Durch eine große individuelle Streuung wird die statistische Sicherung schwachausgeprägter biochemischer Effekte erschwert bzw. unmöglich gemacht. Deshalb sollte das Tiermaterial im Hinblick auf die für das Experiment ausgewählten biochemischen Kenngrößen zuvor auf Homogenität geprüft und im Bedarfsfall ausgewählt werden.

\section{Danksagung}

Für die statistische Auswertung der Ergebnisse danken wir Frl. Füchsel und Herrn Dr. Bräuer.

\section{Literatur}

1. Bloomer, J. R. (1982) Porphyrinmetabolism. In: The Liver: Biology and Pathobiology (Arias, J., Popper, H., Schachter, D. \& Schafritz, D. A., Eds.) Raven Press, New York, pp. $333-345$.

2. Quick, A. J. (1931) J. Biol. Chem. 92, 65-85.

3. Savitzky, A. \& Golay, M. J. E. (1964) Anal. Chem. 36, 1627-1635.

4. Nolte, K. D. \& Gartzke, J. (1985) Z. Med. Lab. Diagn. 26, 402-404.

5. Gartzke, J. \& Weigmann, H.-J. (1979) J. Chromatogr. 162, 234-236.

6. Helger, R., Rindfrey, H. \& Hilgenfeldt, J. (1974) J. Clin. Chem. Clin. Biochem. 12, 344-349.

7. Distlerath, L. M., Larry, D. \& Guengerich, F. P. (1984) Banbury Report 16: Genetic Variability in Responses to Chemical Exposure. Cold Spring Harbor Laboratory, pp. 85-95.

Dr. Joachim Gartzke

Zentralinstitut für

Arbeitsmedizin der DDR

Nöldnerstr. 40/42

DDR-1134 Berlin 
